\title{
Scaling of Mandelbrot Sets Generated by Critical Point Preperiodicity
}

\author{
J.-P. Eckmann ${ }^{\star}$ and H. Epstein
}

Institut des Hautes Etudes Scientifiques, 35, route de Chartres, F-91440 Bures-sur-Yvette, France

\begin{abstract}
Let $z \rightarrow f_{\mu}(z)$ be a complex holomorphic function depending holomorphically on the complex parameter $\mu$. If, for $\mu=0$, a critical point of $f_{0}$ falls after a finite number of steps onto an unstable fixed point of $f_{0}$, then, in the parameter space, near 0 , an infinity of more and more accurate copies of the Mandelbrot set appears. We compute their scaling properties.
\end{abstract}

In several numerical experiments, the dynamics of rational maps has been studied as the coefficients of the map vary. It has been observed [M, DH, S, CGS, C] that remarkably precise copies of the standard Mandelbrot set $\mathscr{M}$ appear in the corresponding plots. In their recent work [DH], Douady and Hubbard explain this phenomenon by the local occurrence of "polynomial-like maps of degree 2." In this paper, we elaborate on this explanation by showing that the mechanism of "quadratification by large order iteration" [G, EEW] produces infinite sequences of copies of $\mathscr{M}$, obeying simple scaling properties.

We describe now the setting of a pre-periodic critical point, but immediately restrict our discussion to the case of period one. We consider a complex holomorphic function $(z, \mu) \rightarrow f_{\mu}(z)$ over $H \times D_{M} \subset \mathbb{C}^{2}$, where $H$ is a domain in $\mathbb{C}$ and $D_{M}=\{\zeta \in \mathbb{C}|| \zeta \mid<M\}, M>0$. We denote by $f_{\mu}$ the function $z \rightarrow f_{\mu}(z)$, and $D^{\alpha \beta} f_{\mu}$ $=\partial_{z}^{\alpha} \partial_{\mu}^{\beta} f_{\mu}{ }^{1}, f_{\mu}^{\prime}=\partial_{z} f_{\mu}$, etc. We make the following assumptions:

A1: $f_{0}$ has a non-degenerate critical point $c$.

A2: $f_{0}$ has an unstable fixed point $u, f_{0}^{\prime}(u)=\gamma,|\gamma|>1$. (The restriction of $f_{0}$ to a sufficiently small neighbourhood of $u$ has a unique inverse which we denote $f_{0}^{-1}$.)

A3: (critical point preperiodicity) $f_{0}^{Q}(c)=u$,

$$
\left(f_{0}^{Q}\right)^{\prime \prime}(c) \neq 0, \text { for some } Q \geqq 1 .
$$

A4: There is a sequence $\left\{x_{n}\right\}$ of points in $H$ accumulating at $u$ such that

$$
f_{0}\left(x_{n+1}\right)=x_{n}, f_{0}^{\prime}\left(x_{n}\right) \neq 0, \text { for } n=1,2, \ldots,
$$

* On leave from the University of Geneva

1 By abuse of notation, we write $D^{\alpha \beta} f_{0}$ instead of $\left.D^{\alpha \beta} f_{\mu}\right|_{\mu=0}$ 
and $x_{0}=c$. Furthermore, for sufficiently large $n$ one has $x_{n}=f_{0}^{-1}\left(x_{n-1}\right)$, with $f_{0}^{-1}$ as in $\mathrm{A} 2$.

If $M$ above is chosen sufficiently small, then $f_{\mu}$ will possess a critical point $c_{\mu}$ and an unstable fixed point $u_{\mu}$ for $\mu \in D_{M}$, depending analytically on $\mu$ and such that $c_{0}=c, u_{0}=u$. We further assume

A 5: $\left.\partial_{\mu}\left[f_{\mu}^{Q}\left(c_{\mu}\right)-u_{\mu}\right]\right|_{\mu=0} \neq 0$.

By the implicit function theorem, reducing $M$ and $H$ somewhat, we may change coordinates in such a way that $c_{\mu}=c, u_{\mu}=u$ for all $\mu \in D_{M}$ and that A1-A5 still hold.

Our result is a scaling relation for $p$-fold iterated functions.

Theorem 1. There are non-zero constants $K_{1}, K_{2}, K_{3}$, and $K$ such that for all sufficiently large $p$ one has:

1. There is a number $\mu_{p}$ for which $f_{\mu_{p}}^{p}(c)=c$ and which satisfies

$$
\left|\mu_{p}-K_{1} \gamma^{-p}\right| \leqq K|\gamma|^{-2 p}
$$

2. The quantity $\lambda_{p} \equiv 2 /\left(f_{\mu_{p}}^{p}\right)^{\prime \prime}(c)$ satisfies

$$
\left|\lambda_{p}-K_{2} \gamma^{-p}\right| \leqq K|\gamma|^{-3 p / 2} .
$$

3. The quantity $\left.\delta_{p} \equiv \lambda_{p}^{-1} \partial_{\mu} f_{\mu}^{p}(c)\right|_{\mu=\mu_{p}}$ satisfies

$$
\left|\delta_{p}-K_{3} \gamma^{2 p}\right| \leqq K|\gamma|^{7 p / 4} \text {. }
$$

4. The function

$$
F_{a}(z ; p) \equiv \lambda_{p}^{-1}\left(f_{\mu_{p}+a / \delta_{p}}^{p}\left(\lambda_{p} z+c\right)-c\right)
$$

satisfies

$$
\left|F_{a}(\zeta ; p)-\zeta^{2}-a\right| \leqq K|\gamma|^{-p / 4}
$$

for all $|\zeta|<|\gamma|^{p / 16} / 2 K_{2}$ and $|a|<|\gamma|^{p / 8}$.

For numerical calculations, it may be useful to give the constants $K_{1}, K_{2}, K_{3}$. One has

$$
\begin{aligned}
& K_{1}=\gamma^{Q} \lim _{n \rightarrow \infty}\left[\gamma^{n}\left(x_{n}-u\right)\right] / \partial_{\mu} f_{0}^{Q}(c), \\
& K_{2}=2 \gamma^{Q} /\left(\lim _{n \rightarrow \infty}\left[\left(f_{0}^{n}\right)^{\prime}\left(x_{n}\right) \gamma^{-n}\right] \cdot\left(f_{0}^{Q}\right)^{\prime \prime}(c)\right), \\
& K_{3}=2 \partial_{\mu} f_{0}^{Q}(c) /\left(K_{2}^{2}\left(f_{0}^{Q}\right)^{\prime \prime}(c)\right) .
\end{aligned}
$$

As a result of the theorem, the sequence of functions $(\zeta, a) \rightarrow F_{a}(\zeta ; p)$ tends, as $p \rightarrow \infty$, uniformly on every compact set to $(\zeta, a) \rightarrow P_{a}(\zeta) \equiv \zeta^{2}+a$. For any $A \geqq 4,|a| \leqq A$, we have $P_{a}^{-1}\left(D_{A^{2}}\right) \subset D_{2 A}$. Hence, for $r>2 A$ and sufficiently large $p$, we have $F_{a}^{-1}\left(D_{A^{2}} ; p\right) \subset D_{r}$ and the triple $\left(D_{A^{2}}, F_{a}^{-1}\left(D_{A^{2}} ; p\right), F_{a}(\cdot ; p)\right)$ is a "polynomial-like map of degree 2 " in the sense of Douady and Hubbard [DH]. Recall that the Mandelbrot set $\mathscr{M}$ of the family $\left\{P_{a}\right\}$ is given by

$$
\mathscr{M}=\bigcap_{n=1}^{\infty} g_{n}^{-1}\left(D_{A^{2}}\right), \text { where } g_{n}(a)=P_{a}^{n}(0) .
$$


Clearly, the local Mandelbrot set $\mathscr{M}_{p}$ of the family $\left\{F_{a}(\cdot ; p)\right\}$, similarly defined, converges to $\mathscr{M}$ as $p \rightarrow \infty$. In particular,

$$
\mathscr{M}^{c} \subset \bigcup_{R \geqq 1} \bigcap_{p \geqq R} \mathscr{M}_{p}^{c}
$$

On the other hand, $\mathscr{M}$ contains the open set $\mathscr{M}^{\prime}=\left\{a \mid P_{a} \text { has an attractive cycle }\right\}^{2}$. If $a \in \mathscr{M}^{\prime}$, then, for sufficiently large $p, F_{a}(\cdot ; p)$ has an attractive cycle of the same period as $P_{a}$ which, by the results of [DH], attracts the critical point 0 . Thus, if we define $\mathscr{M}_{p}^{\prime}$ in analogy with $\mathscr{M}^{\prime}$, we find

$$
\mathscr{M}^{\prime} \subset \bigcup_{R \geqq 1} \bigcap_{p \geqq R} \mathscr{M}_{p}^{\prime}
$$

Theorem 1, combined with (5), (6) shows the asserted accumulation of Mandelbrot sets near $\mu=0$, and their scale and position.

Before we start with the proof of the theorem proper, we restate A1-A5 and some of their consequences as $\mathrm{H} 1-\mathrm{H} 4$ below.

There are positive constants $L, M, U<1, R\left(=8+16 L|\gamma| /\left(1-|\gamma|^{-3 / 5}\right)\right)$, such that:

H1: For $\mu \in D_{M}, f_{\mu}$ has a non-degenerate critical point at $c$.

$\mathrm{H} 2$ : For all $\mu \in D_{M}$, the restriction of $f_{\mu}$ to $u+D_{U}$ has a unique inverse $f_{\mu}^{-1}$, which satisfies for all $z \in u+D_{U}$ :

1. $f_{\mu}^{-1}(z) \in u+D_{U},\left|\left(f_{\mu}^{-1}\right)^{\prime}(z)\right| \leqq|\gamma|^{-4 / 5}$.

2. $\left|f_{\mu}^{-1}(z)-u-\gamma^{-1}(z-u)\right| \leqq L|z-u|(|z-u|+|\mu|)$.

3. $\mid\left(f_{\mu}^{\prime}(z)-\gamma \mid \leqq L(|z-u|+|\mu|)\right.$. In particular, $\left|f_{\mu}^{\prime}(z)\right| \leqq|\gamma|^{5 / 4}$.

4. $\left|\partial_{\mu} f_{\mu}(z)\right| \leqq L|z-u|$.

H3: There is a $P \geqq 1$ and a holomorphic function $\mu \rightarrow z_{\mu}$ from $D_{M}$ to $u+D_{U / R}$, such that for all $\mu \in D_{M}$ one has

1. $f_{\mu}^{P}\left(z_{\mu}\right)=c$.

2. $\left|D^{\alpha \beta} f_{\mu}^{P}(z)-D^{\alpha \beta} f_{0}^{P}\left(z_{0}\right)\right| \leqq L\left(\left|z-z_{0}\right|+|\mu|\right), \forall \alpha+\beta \leqq 3, \forall z \in u+D_{U}$.

3. $\left|\left(f_{\mu}^{P}\right)^{\prime}\left(z_{\mu}\right)\right|>L^{-1},\left|\partial_{\mu} f_{0}^{P}\left(z_{0}\right)\right| \leqq L$.

4. $\left|z_{\mu}-u\right| \geqq L^{-1}$.

5. $x_{P}=z_{0}, x_{P+n}=f_{0}^{-n}\left(x_{P}\right)$ for $n \geqq 0, x_{P-n}=f_{0}^{n}\left(x_{P}\right)$ for $0 \leqq n \leqq P$.

H4: There is a $C>0$ such that for every $\mu \in D_{M}$, one has

1. $\left|D^{\alpha \beta} f_{\mu}^{Q}(z)-D^{\alpha \beta} f_{0}^{Q}(c)\right| \leqq L(|z-c|+|\mu|), \forall \alpha+\beta \leqq 3, \forall z \in c+D_{C}$.

2. $f_{0}^{Q}(c)=u,\left|\partial_{\mu} f_{\mu}^{Q}(c)\right|>L^{-1}$.

3. $\left|\left(f_{\mu}^{Q}\right)^{\prime \prime}(c)\right|>L^{-1}$.

Proof of Theorem 1.1 (Well-known: see e.g. [G], [DH]). By H4.2, we see that for sufficiently small $M,\left\{f_{\mu}^{Q}(c)|| \mu \mid=M / 2\right\}$ is a contour around $u$. By $\mathrm{H} 2.2$ and $\mathrm{H} 3$, $f_{\mu}^{-s}\left(z_{\mu}\right)$ lies inside this contour for sufficiently large $s$. Thus, by Rouché's theorem, there is a unique $\mu$ in $D_{M / 2}$ for which $f_{\mu}^{Q}(c)=f_{\mu}^{-s}\left(z_{\mu}\right)$, i.e. $f_{\mu}^{s+P+Q}(c)=c$. Thus we have found $\mu_{s+P+Q}$. For a quantitative treatment, we define, for all $(z, \mu) \in\left(u+D_{U}\right)$ $\times D_{M}$,

$$
h_{\mu}(z)=\lim _{n \rightarrow \infty} \gamma_{\mu}^{n}\left(f_{\mu}^{-n}(z)-u\right)
$$

2 We have no result concerning $\mathscr{\mathscr { M }} \backslash \mathscr{M}^{\prime}$, the so-called "composantes farfelues" [DH, MSS, ST] 
where $\gamma_{\mu}=f_{\mu}^{\prime}(u)$. By H2.2, the above limit exists uniformly in $z, \mu$ and satisfies

$$
h_{\mu}(u)=0, h_{\mu}^{\prime}(u)=1, h_{\mu}\left(f_{\mu}^{-1}(z)\right)=\gamma_{\mu}^{-1} h_{\mu}(z) \text {. }
$$

We can now rewrite $f_{\mu}^{s+P+Q}(c)=c$ as

$$
\gamma_{\mu}^{-s}=h_{\mu}\left(f_{\mu}^{Q}(c)\right) / h_{\mu}\left(z_{\mu}\right) \equiv v(\mu) .
$$

Since $z_{0} \neq u$ by H3.4, we see from H3.2 and (6) that $v$ is defined and $v^{\prime}(0) \neq 0$. Using again $\mathrm{H} 2.2$, we see that

$$
\mu=\gamma^{-s}\left(1+\mu s k_{s}(\mu)\right) / v^{\prime}(0),
$$

with $k_{s}(\mu)$ uniformly bounded for large $s$ and $\mu \in D_{M / 2}$. Theorem 1.1 follows by the contraction mapping theorem. A little algebra shows (2) in the form

$$
K_{1}=\gamma^{Q} h_{0}\left(x_{0}\right) / \partial_{\mu} f_{0}^{Q}(c), h_{0}\left(x_{0}\right) \equiv \gamma^{P} h_{0}\left(x_{P}\right) .
$$

As a preparation for the proof of Theorem 1.2-1.4, we study the deviation of a function $\varphi$ from a quadratic polynomial by considering ([G, EEW]) the magnitude of $\varphi^{\prime \prime} / \varphi^{\prime}-1 / z$. We therefore set, for large $p$,

$$
\Delta_{\mu}(z ; p)=\frac{\left(f_{\mu}^{p}\right)^{\prime \prime}(z)}{\left(f_{\mu}^{p}\right)^{\prime}(z)}-\frac{\left(f_{\mu}^{Q}\right)^{\prime \prime}(z)}{\left(f_{\mu}^{Q}\right)^{\prime}(z)} .
$$

Lemma 2. There is a constant $K$ such that for sufficiently large $p$ and all

$$
z \in c+D_{|\gamma|^{-3 p / 4}}, \mu \in \mu_{p}+D_{|\gamma|^{-3 p / 2}}
$$

the inequality

$$
\left|\Delta_{\mu}(z ; p)\right|<K\left|\left(f_{\mu}^{Q}\right)^{\prime}(z)\right||\gamma|^{p}
$$

holds.

Proof. We set $s=p-P-Q$. Let $|\zeta-u|<2 U / R<U<1,|\mu|<2 K_{1}|\gamma|^{-p}$. By H2, $\left|f_{\mu}^{-n}(\zeta)-u\right| \leqq|\gamma|^{-4 n / 5}|\zeta-u|$. By induction, using $\mathrm{H} 2.2$, for all $n=0,1, \ldots, s$,

$$
\left|f_{\mu}^{-n}(\zeta)-u\right| \leqq|\gamma|^{-n}|\zeta-u|\left[1+L|\gamma|\left(|\zeta-u|+2 K_{1}|\gamma|^{-p / 5}\right) \frac{1-|\gamma|^{-3 n / 5}}{1-|\gamma|^{-3 / 5}}\right] \text {. }
$$

For sufficiently large $p$, this gives, by the definition of $R$,

$$
\left|f_{\mu}^{-n}(\zeta)-u\right|<2|\gamma|^{-n} \frac{U}{R}\left[1+2 L|\gamma| /\left(1-|\gamma|^{-3 / 5}\right)\right] \leqq|\gamma|^{-n} U / 4
$$

We now fix $z$ and $\mu$ satisfying (9) and denote, for $0 \leqq n \leqq s$,

$$
w=f_{\mu}^{Q}(z), \hat{w}=f_{\mu_{p}}^{Q}(c), \hat{w}_{n}=f_{\mu_{p}}^{n}(\hat{w})=f_{\mu_{p}}^{n-s}\left(z_{\mu_{p}}\right) .
$$

By the above estimate and Theorem 1.1,

$$
\left|\hat{w}_{n}-u\right|<|\gamma|^{n-s} U / 4 \text {. }
$$

By (9), $|w-\hat{w}| \leqq 2 L|\gamma|^{-3 p / 2}$ and $|w-u|<U / 2$. An induction on $n, 1 \leqq n \leqq s$, shows that $w_{n} \equiv f_{\mu}^{n}(w)$ is defined, and

$$
\begin{aligned}
\left|w_{n}-\hat{w}_{n}\right| & \leqq 2 L\left|\mu-\mu_{p}\right|+|\gamma|^{5 / 4}\left|w_{n-1}-\hat{w}_{n-1}\right| \\
& \leqq 2 L|\gamma|^{5 n / 4-3 p / 2}\left(1-|\gamma|^{-5(n+1) / 4}\right) /\left(1-|\gamma|^{-5 / 4}\right) .
\end{aligned}
$$


This implies $\left|w_{n}-u\right|<U / 2,\left|w_{s}-u\right|<2 U / R$, for sufficiently large $p$, since $\left|\hat{w}_{s}-u\right|$ $<U / R$. The first estimate, with $\zeta=w_{s}$, now gives:

$$
\left|w_{n}-u\right|<|\gamma|^{n-s} U / 4, \quad 0 \leqq n \leqq s .
$$

We can now turn to

$$
\Delta_{\mu}(z ; p)=\left(f_{\mu}^{Q}\right)^{\prime}(z)\left\{\frac{\left(f_{\mu}^{P}\right)^{\prime \prime}\left(w_{s}\right)}{\left(f_{\mu}^{P}\right)^{\prime}\left(w_{s}\right)} \cdot\left(f^{s}\right)^{\prime}(w)+\frac{\left(f^{s}\right)^{\prime \prime}(w)}{\left(f^{s}\right)^{\prime}(w)}\right\} .
$$

By H3, we have

$$
\left|\left(f_{\mu}^{P}\right)^{\prime \prime}(\zeta) /\left(f_{\mu}^{P}\right)^{\prime}(\zeta)\right| \leqq L^{2}, \quad \forall \zeta \in u+D_{U},
$$

while $\mathrm{H} 2$ implies

$$
\left|\left(f_{\mu}^{j}\right)^{\prime}(w)\right|=\prod_{k=0}^{j-1}\left|f_{\mu}^{\prime}\left(w_{k}\right)\right| \leqq|\gamma|^{j} \prod_{k=0}^{j-1}\left(1+\mathcal{O}\left(|\gamma|^{-(k-s) / 4}\right)\right) \leqq \mathcal{O}\left(|\gamma|^{j}\right) .
$$

Since

$$
\frac{\left(f_{\mu}^{s}\right)^{\prime \prime}(w)}{\left(f_{\mu}^{s}\right)^{\prime}(w)}=\sum_{j=0}^{s-1} \frac{f_{\mu}^{\prime \prime}\left(w_{j}\right)}{f_{\mu}^{\prime}\left(w_{j}\right)}\left(f^{j}\right)^{\prime}(w)
$$

we find

$$
\left|\left(f_{\mu}^{s}\right)^{\prime \prime}(w) /\left(f_{\mu}^{s}\right)^{\prime}(w)\right| \leqq \mathcal{O}\left(|\gamma|^{s}\right),
$$

and Lemma 2 follows by combining (13)-(16).

For later use, we note that (12) describes accurately the position of $w_{n}$.

Proof of Theorem 1.2. By definition, we have $2 \lambda_{p}^{-1}=\left(f_{\mu_{p}}^{p}\right)^{\prime \prime}(c)$, which we decompose as

$$
\left(f_{\mu_{p}}^{p}\right)^{\prime \prime}(c)=\left(f_{\mu_{p}}^{P}\right)^{\prime}\left(\hat{w}_{s}\right) \cdot\left(f_{\mu_{p}}^{s}\right)^{\prime}(\hat{w}) \cdot\left(f_{\mu_{p}}^{Q}\right)^{\prime \prime}(c),
$$

with the notations of the proof of Lemma 2. Using now (11) and $\mathrm{H} 2$ to control $\left(f_{\mu_{p}}^{s}\right)^{\prime}$ and $\mathrm{H} 4$ to control $\left(f_{\mu_{p}}^{s}\right)^{\prime}$ and $\left(f_{\mu_{p}}^{Q}\right)^{\prime \prime}$, one derives immediately Theorem 1.2, and a simple calculation leads to (3), in the form

$$
K_{2}=2 \gamma^{Q} h_{0}^{\prime}\left(x_{0}\right) /\left(f_{0}^{Q}\right)^{\prime \prime}(c), h_{0}^{\prime}\left(x_{0}\right) \equiv \gamma^{P} h_{0}^{\prime}\left(x_{P}\right) /\left(f_{0}^{P}\right)^{\prime}\left(x_{P}\right) .
$$

Note that ( $\left.2^{\prime}\right)$ and $\left(3^{\prime}\right)$ may be more convenient for numerical work than (2) and (3), since $h_{0}$ can be computed from its Taylor series.

We define

$$
H_{\mu}(\zeta ; p)=\lambda_{p}^{-1} f_{\mu}^{P}\left(\lambda_{p} \zeta+c\right)
$$

Lemma 3. For large $p$ and for

$$
\zeta \in D_{|\gamma| p / 4 / 2 K_{2}}, \quad \mu \in \mu_{p}+D_{|\gamma|^{-3 p / 2}}
$$

(where $K_{2}$ is defined by Theorem 1.2), one has

$$
\left|H_{\mu}(\zeta ; p)-H_{\mu}(0 ; p)-H_{\mu}^{\prime \prime}(0 ; p) \zeta^{2} / 2\right| \leqq\left|H_{\mu}^{\prime \prime}(0 ; p)\right| \mathcal{O}\left(|\zeta|^{3}|\gamma|^{-3 p / 4}\right) .
$$


Proof. Denote

$$
\begin{aligned}
G_{\mu}(\zeta ; p) & =H_{\mu}^{\prime \prime}(\zeta ; p) / H_{\mu}^{\prime}(\zeta ; p)-1 / \zeta, \\
g(z, \mu) & =\left(f_{\mu}^{Q}\right)^{\prime \prime}(z) /\left(f_{\mu}^{Q}\right)^{\prime}(z)-1 /(z-c) .
\end{aligned}
$$

Then

$$
G_{\mu}(\zeta ; p)=\lambda_{p} \cdot \Delta_{\mu}\left(\lambda_{p} \zeta+c ; p\right)+\lambda_{p} g\left(\lambda_{p} \zeta+c, \mu\right)
$$

Our choice of domain, Eq. (18), assures that by Lemma 2,(20) is defined. Note that by $\mathrm{H} 4,\left|g\left(\lambda_{p} \zeta+c, \mu\right)\right|<2 L^{2}$, and hence by Lemma 2 and (17),

$$
\left|G_{\mu}(\zeta ; p)\right| \leqq \mathcal{O}\left(|\gamma|^{-3 p / 4}\right) \text { for } \zeta, \mu \text { as in (18). }
$$

Since $G_{\mu}(\zeta ; p)=\partial_{\zeta} \log \left(H_{\mu}^{\prime}(\zeta ; p) / \zeta\right)$ and $H_{\mu}^{\prime}(\zeta ; p) /\left.\zeta\right|_{\zeta=0}=H_{\mu}^{\prime \prime}(0 ; p)$, we can integrate, and we get

$$
H_{\mu}^{\prime}(\zeta ; p)=\zeta H_{\mu}^{\prime \prime}(0 ; p) \exp \int_{0}^{\zeta} G_{\mu}(\eta ; p) d \eta
$$

Hence,

$$
\left|H_{\mu}^{\prime}(\zeta ; p)-\zeta H_{\mu}^{\prime \prime}(0 ; p)\right| \leqq \mathcal{O}\left(\left|\zeta^{2} H_{\mu}^{\prime \prime}(0 ; p)\right||\gamma|^{-3 p / 4}\right) ;
$$

and the assertion follows by an additional integration.

Proof of Theorem 1.3. We want to relate the parametrization of $H_{\mu}$ to that given by the quantity $H_{\mu}(0 ; p)$. For this purpose, we study $\partial_{\mu} f_{\mu}^{p}(z)$, with $z=\lambda_{p} \zeta+c$, and $\zeta, \mu$ as in (18). We rewrite

$$
\partial_{\mu} f_{\mu}^{p}(z)=\left(\partial_{\mu} f_{\mu}^{P}\right)\left(w_{s}\right)+\left(f_{\mu}^{P}\right)^{\prime}\left(w_{s}\right) \cdot\left(\partial_{\mu} f_{\mu}^{s}\right)\left(w_{0}\right)+\left(f_{\mu}^{P}\right)^{\prime}\left(w_{s}\right) \cdot\left(f_{\mu}^{s}\right)^{\prime}\left(w_{0}\right) \partial_{\mu} f_{\mu}^{Q}(z),
$$

with $w_{s}=f_{\mu}^{s+Q}(z)$. We shall see that the last term in (21) dominates, and we therefore start by majorizing the first two terms.

By H3, and our earlier bounds (12) on $w_{s}$, we have

$$
\left|\partial_{\mu} f_{\mu}^{P}\left(w_{s}\right)\right| \leqq C_{1}
$$

with $C_{1}$ independent of $p, s, \mu, z$. By $\mathrm{H} 2$, we may write

$$
\left(\partial_{\mu} f_{\mu}^{s}\right)\left(w_{0}\right)=\sum_{n=0}^{s-1}\left(f_{\mu}^{n}\right)^{\prime}\left(w_{s-n}\right)\left(\partial_{\mu} f_{\mu}\right)\left(w_{s-n-1}\right) .
$$

Using (12) and $\mathrm{H} 2.4$, we see that

$$
\left|\partial_{\mu} f_{\mu}^{s}\left(w_{0}\right)\right| \leqq \sum_{n=0}^{s-1} C_{2}|\gamma|^{n}\left|w_{s-n-1}-u\right| \leqq p \cdot C_{3}|\gamma|^{n}|\gamma|^{-n}
$$

Thus the second term in (21) is bounded by a constant multiple of $p$. Using H4.1,2, $\mathrm{H} 2.3$, and $\mathrm{H} 3.2$, we see that

$$
\left(f_{\mu}^{\boldsymbol{P}}\right)^{\prime}\left(w_{s}\right)\left(f_{\mu}^{s}\right)^{\prime}\left(w_{0}\right) \partial_{\mu} f_{\mu}^{Q}(z)=\left(\left(f_{0}^{P}\right)^{\prime}\left(z_{0}\right) / h_{0}^{\prime}\left(z_{0}\right)\right) \gamma^{p-P-Q} \partial_{\mu} f_{0}^{Q}(c)\left(1+R|\gamma|^{-p / 4}\right),
$$


with $R$ bounded independently of $p, s, \mu, z$. This asymptotic behavior applies now to $\partial_{\mu} f_{\mu}^{p}$ as well and Theorem 1.3 follows at once. Again, we leave (4) as an exercise.

Proof of Theorem 1.4. We have just seen that

$$
\left.\delta_{p} \equiv \lambda_{p}^{-1} \partial_{\mu} f_{\mu}^{p}(c)\right|_{\mu=\mu_{p}}=K_{3} \gamma^{2 p}\left(1+\mathcal{O}\left(|\gamma|^{-p / 4}\right) .\right.
$$

Using the definition of $H_{\mu}$, we get from Theorem 1.3, that

$$
\left|\partial_{\mu} H_{\mu}(\zeta ; p)\right| \leqq C_{5}|\gamma|^{2 p} \text {, for } \zeta, \mu \text { as in (18). }
$$

By the Cauchy inequalities, this implies

$$
\left|\partial_{\mu} H_{\mu}^{\prime \prime}(0 ; p)\right| \leqq 2 C_{5}|\gamma|^{2 p} /|\gamma|^{p / 2}=2 C_{5}|\gamma|^{3 p / 2},
$$

and hence

$$
\left|H_{\mu}^{\prime \prime}(0 ; p)-2\right| \leqq 2 C_{5}|\gamma|^{3 p / 2}\left|\mu-\mu_{p}\right| .
$$

If we require now $\left|\mu-\mu_{p}\right|<|\gamma|^{-15 p / 8}$, then

$$
\left|H_{\mu}(0 ; p)-\delta_{p}\left(\mu-\mu_{p}\right)\right| \leqq C_{6}|\gamma|^{2 p}\left|\mu-\mu_{p}\right|^{2} /\left(|\gamma|^{-3 p / 2}-|\gamma|^{-15 p / 8}\right) \leqq \mathcal{O}\left(|\gamma|^{-p / 4}\right) .
$$

Combining (23), (24) with Lemma 3, we get Theorem 1.4.

\section{References}

[C] Curry, J.: On the periodic behavior of Newton's method for a family of real cubic polynomials. Preprint, Boulder

[CGS] Curry, J., Garnett, L., Sullivan, D.: On the iteration of a rational function: computer experiments with Newton's method. Commun. Math. Phys. 91, 267 (1983)

[DH] Douady, A., Hubbard, J.: In preparation; CRAS t. 294

Douady, A.: Dynamique des applications à allure polynômiale, exposé à la RCP 25, Strasbourg 1982

Douady, A.: Systèmes dynamiques holomorphes. Sém. Bourbaki No. 599, 1982

[EEW] Eckmann, J.-P., Epstein, H., Wittwer, P.: Fixed points of Feigenbaum type for the equation $f^{p}(\lambda x)=\lambda f(x)$. Commun. Math. Phys. 93, 495 (1984)

[G] Guckenheimer, J.: Draft, University of California, Santa Cruz (1983); Commun. Math. Phys. 70, 133 (1979)

[M] Mandelbrot, B.: Fractal aspects of the iteration of $z \rightarrow \lambda z(1-z)$ for complex $\lambda$ and $z$. Ann. N. Y. Acad. Sci. 357, 249-259 (1980)

[MSS] Mañé, R., Sad, P., Sullivan, D.: On the dynamics of rational maps. Ann. E.N.S. (to appear)

[ST] Sullivan, D., Thurston, W.: Note on structural stability of rational maps. Preprint IHES, 1982

[S] Sullivan, D.: Quasi-conformal homeomorphisms and dynamics. I, II, III. IHES preprints 1982,1983

Communicated by O. E. Lanford

Received October 24, 1983

Note added in proof. We have learned from A. Douady that he and J. Hubbard have obtained the same results, which will appear in their paper in preparation. 
